\title{
L'institutionnalisation d'une équipe de recherche en S.T.A.P.S. : un analyseur transactionnel
}

Yves Le Pogam

\section{(2) OpenEdition}

1 Journals

Édition électronique

URL : http://journals.openedition.org/corpsetculture/284

DOI : 10.4000/corpsetculture.284

ISSN : $1777-5337$

Éditeur

Association Corps et Culture

Édition imprimée

Date de publication : 1 juin 1995

ISSN : 1268-5631

Référence électronique

Yves Le Pogam, «L'institutionnalisation d'une équipe de recherche en S.T.A.P.S. : un analyseur

transactionnel », Corps et culture [En ligne], Numéro 1 | 1995, mis en ligne le 24 janvier 2017, consulté le 08 septembre 2020. URL : http://journals.openedition.org/corpsetculture/284 ; DOI : https://doi.org/ 10.4000 /corpsetculture.284

Ce document a été généré automatiquement le 8 septembre 2020

(c) tous droits réservés 


\title{
L'institutionnalisation d'une équipe de recherche en S.T.A.P.S. : un analyseur transactionnel
}

\author{
Yves Le Pogam
}

1 La création d'une équipe de recherche est toujours un événement dont il serait faux de croire qu'il n'a pas déjà une histoire. Comme tel, il devient nécessaire de le considérer comme un analyseur permettant de révéler les enjeux liés à une double dynamique. Dynamique interne d'abord, car il s'agit de réguler et de fédérer les points de vue différents sur les attentes de la recherche des membres qui constituent une équipe en création, externe ensuite, car cette dernière doit se positionner dans un établissement où la recherche est déjà structurée et doit se singulariser dans l'ensemble des recherches en S.T.A.P.S portées par des champs disciplinaires proches ou différents, générant des points de vue variés sur ce qu'est ou devrait être la recherche. C'est bien de ces différentes "transactions" (Rémy-Voyé) entre le dedans et le dehors que naît l'événement, produit par cet ensemble de contraintes de compréhension régulant les interactions entre les acteurs, lié aux enjeux et toujours surplombé par la nécessité d'allégeance à une scientificité garantie par les sociétés de discours, ici le conseil scientifique de l'UFR. Il faut donc renoncer à considérer cette institutionnalisation comme une création "spontanée", un jaillissement pulsionnel ou un surgissement imprévisible, et plutôt refroidir l'événement en l'envisageant comme le résultat d'un dépassement de contraintes qui a nécessité dialogues, mises au point, défenses argumentées, dont l'objectif visait moins à établir un consensus entre les membres de l'équipe, qu'à légitimer leur reconnaissance dans un champ déjà structuré en deux équipes. L'intégration d'une troisième dimension dans un laboratoire de recherche constitué, entraîne la recomposition de l'ensemble et impose de nouvelles formes de régulation des pouvoirs ainsi que de nouveaux apprentissages de vie collective, longs à acquérir, produits par un changement dont les effets sont toujours imprévisibles puisqu'ils engagent des acteurs mus par la divergence de leurs intérêts et de leurs interprétations sur les attendus de la recherche. 
2 Quatre années se sont donc écoulées entre les premiers désirs de reconnaissance de ce nouveau groupe et sa prise en compte effective par une institution légitimante. Il faut donc s'abstraire de la croyance naïve en une possible édification d'une structure de recherche jaillie de la passion ou d'une curiosité louable, pour l'objectiver dans un réseau de contraintes et de transactions raisonnées, légitimées par un désir d'institutionnalisation: passion et émotion y sont transfigurées par l'adéquation à la norme.

3 Cette lente gestation tient d'abord à une cause interne : la mémoire institutionnelle. Structurée déjà en deux équipes unifiées par le label "Jeune équipe DRED", organisée autour de l'école doctorale, l'assise de la recherche dans l'institution était certes ouverte à la reconnaissance d'une troisième force, à condition bien sûr qu'elle souscrive à la garantie de scientificité, en l'occurrence qu'elle fasse émerger des problèmes nettement définis qui nécessitent une recherche organisée autour d'une thématique exploitée selon des normes méthodologiques adéquates, faisant l'objet de publications reconnues et expertisées par des comités de lecture garants de leur adéquation à la norme. Cette proximité de pairs confirmés par la qualification de "Jeune équipe DRED", induisait de la part des demandeurs une référence au procès de scientificité certes, mais générait en même temps- et peut-être paradoxalement- une volonté de distanciation: le champ des sciences sociales qui composait l'essence du groupe ne pouvait se conformer, épistémologiquement, aux critères retenus. Le groupe allait donc vivre un dilemme et se trouver dans une situation schizophrénique née de l'obligation de souscrire à un système référentiel extérieur, sans cependant rompre avec sa revendication d'autonomie qu'il considérait comme une clause de sauvegarde tenant à sa spécificité. Cette situation "crisique" pouvait être facilement résolue par l'adéquation consensuelle à la praxis scientifique qui accorderait l'ensemble des chercheurs par leur adhésion à cette norme commune, mais ce serait laisser croire que la science ne se positionne que sur des critères de rigueur de la preuve. Chacun sait que ces critères obéissent aussi à une raison "disputationnelle" (Habermas), dont les enjeux sont sociaux et conduisent à considérer la "vérité" comme le produit de négociations, de communications, voire dans notre langage, de transactions, qui refoulent l'idée d'une raison purement objective comme surdimensionnant le processus de légitimation de la recherche. La raison scientifique combine la logique d'adéquation à la référence garantie par les sociétés de discours- logique rationaliste - à une logique transactionnelle, c'est-à-dire raisonnée, produite par le jeu régulateur des échanges entre les acteurs. Cette "raison discursive" (Habermas) produite par l'adhésion de la communauté à certaines règles argumentatives, conduisait les demandeurs à de nécessaires rencontres lors de séminaires, dont l'objectif était d'identifier les spécificités de chacun des membres de l'équipe persuadés qu'il existait bien une unité épistémologique dans le groupe, dans le but de parvenir à un titre les identifiant sans ambiguïté, rendant compte en outre de cette pertinence et sur lequel se grefferaient des recherches organisées autour d'axes permettant de réunir certains membres de l'équipe.

4 Après plusieurs tentatives rejetées par le conseil scientifique, le premier titre LABELS (Laboratoire d'étude du loisir sportif) avait été présenté dans une vision trop appliquée, le second LEPAPS (Laboratoire d'études et de prospective en activités physiques et sportives), n'identifiait pas suffisamment une thématique de recherche, l'équipe fut reconnue sous l'appellation de "Corps et culture". Ce titre symbolique, puisqu'il a 
marqué une collection dirigée par Michel Bernard dont certains ouvrages ont fait grand bruit dans le monde des S.T.A.P.S et ailleurs, marque certainement de son autorité bon nombre de membres du groupe, et s'explique aussi parce qu'il rassemble l'équipe, en même temps qu'il ressemble aux choix des titres des deux autres équipes de l'établissement, identifiées non par des champs disciplinaires, mais par une thématique de recherche, même si les trois équipes actuelles agrègent des diversités disciplinaires en leur sein (anthropologie, ethnologie, histoire, sociologie, sciences de l'éducation pour notre groupe, psychologie pour les deux autres).

Cette logique multidisciplinaire, encore imparfaite - puisque les sensibilités dominantes des deux autres groupes sont psychologiques et la nôtre proche des sciences sociales est cependant inévitable pour tenter de faire communiquer autour d'un même objet, des champs disciplinaires multidimensionnels capables d'éclairer les problèmes posés par le corps en mouvement dans des contextes culturels en mutation, permettant d'éviter ce que Edgar Morin appelle "l'école du deuil". En effet, une certaine idée "noble" de la recherche, d'autant plus partagée par la communauté qu'elle s'exerce dans un champ comme celui des S.T.A.P.S qui a besoin de se légitimer par rapport aux attendus des instituts d'excellence de la recherche en France, tend au cloisonnement disciplinaire perturbant la lecture obligatoirement multiréférentielle (Adorno, Brohm) d'une corporéité perçue dans la complexité, dont le sens ne peut être approché que dans l'antagonisme. Cette "école du deuil" dont tendent à être victimes les S.T.A.P.S au nom de la conformité à une vision dominante de la science, oblige les chercheurs souvent pour des raisons carriéristes - à se positionner dans des champs disciplinaires étroits, et cette conformisation brise toutes les audaces, marginalise la frange, concrétise le standard et dirige l'autonomie de la recherche. Les sciences doivent toujours être attentives (et tout particulièrement les sciences sociales) à leur indépendance et une science émancipatrice "requiert de mettre fin aux effets de domination qui distordent la compétition scientifique comme l'élimination de certains qui sont dignes d'entrer dans le jeu (...) ; ou comme la censure qu'exerce la bienséance académique en obligeant les esprits les plus novateurs à dépenser une part considérable de leur temps à fournir les preuves les plus complètes conformément aux canons positivistes du moment, de chacune de leurs propositions, les empêchant ainsi de produire une quantité de propositions nouvelles dont la validation complète pourrait être laissée à d'autres" (Bourdieu, Réponses: 163-164). L'institutionnalisation d'une équipe est bien un analyseur transactionnel, mais qui en aucun cas n'évacue les conflits, il n'est pas consensuel.

Alors, il fallait que la nouvelle équipe se prononce sur un point sensible qui accorde les deux autres équipes et d'une manière plus générale, le champ des S.T.A.P.S : celui de l'utilité de la recherche dont la nécessité si on la comprend dans un sens étroit, n'était pas acquise pour quelques-uns des membres de l'équipe en constitution, soucieux dans un domaine comme celui des sciences sociales, de conserver une liberté de connaissance qui ne soit pas vassalisée à ce qui a priori pouvait vite apparaître comme un utilitarisme étroit et clos, soumis à l'obligation de servir. De quelle utilité parlionsnous? Comment concilier des recherches résolument universitaires avec des commandes sociales? Quelle part accorder à ceux qui ne peuvent concevoir la production que dans une perspective "appliquée" ? N'est-il pas possible, au terme d'une communication dans le groupe, de dépasser - sans qu'il y ait consensus - le classique conflit entre le fondamental et l'appliqué ? Les recherches en S.T.A.P.S dans leur jeune développement peuvent-elles se permettre de condamner l'une ou l'autre direction? 
Ici se pose encore la prise en compte de critères d'indépendance et de réflexivité liés à une science indépendante.

7 Cet ensemble de questions génère encore de nouvelles transactions justifiées par des proximités scientifiques et par l'inconscient structurant la globalité des S.T.A.P.S. Car ici les extrêmes sont a priori disqualifiés par la communauté scientifique : le chercheur isolé parce qu'il est souvent militant et comme tel paraît ne pas présenter des garanties d'objectivité, et les recherches appliquées puisque l'obtention des contrats est parfois prioritaire par rapport à une logique de connaissance dont l'inféodation à un organisme ne serait pas une garantie de la validité des résultats. L'utilité paraît alors incompatible avec le procès de scientificité et conduit soit à des renoncements de contrats toujours "impurs" et à des repliements universitaires à l'abri du soupçon. Mais ces remarques méritent quelques développements car, ici aussi, c'est en terme de transaction qu'il faut analyser les processus.

8 Il a été dit que la recherche est scientifique et sociale, la scientificité ne s'acquiert que par l'analyse des conditions sociales de production de la connaissance, que celle-ci soit appliquée ou fondamentale, et implique alors des dialogues entre le chercheur isolé ou l'équipe des chercheurs et la communauté scientifique. L'utilité de la recherche ne vaut que si les thèmes ne se fixent pas systématiquement par les demandeurs consommateurs de science, et que soient analysées avec autant de perspicacité les recherches "qui marchent bien" souvent vouées à l'application aveugle et celles "qui ne servent à rien" souvent attribuées à l'université (sur toutes ces questions, cf. "Recherches sociologiques et demandes sociales", Revue de l'Institut de Sociologie, Université libre de Bruxelles, 1-2, 1988). Accepter la "raison disputationnelle", c'est analyser les positions axiologiques bien définies qui fondent ces divergences, et dans ce sens, la raison d'utilité vaut autant pour le développement de recherches critiques productrices de connaissance que pour les commandes de décideurs, dès lors que celles-ci ne répondent pas par des réassurances sur les marchés. Ces intérêts multiples qui composent un groupe épistémologiquement hétérogène, poussent ses membres à une constante compréhensivité réciproque produite par des interactions liées aux enjeux axiologiquement définis et indépassables car constamment reformulés par les désordres provoqués par les contextes qui les affectent et les transforment. Entre deux types d'orientations qui caractérisent parfois les recherches en sciences sociales - peutêtre trop facilement dichotomiques - une orientation "d'arrangement" et de "dérangement" ou encore une perspective "de service" et de "connaissance", l'équipe s'oriente vers la seconde, celle qui espère montrer "ce que les sociétés ne se donnent pas à voir en surface" (Balandier), ceci en respectant les différentes théories et procédures méthodologiques auxquelles chaque membre qui la compose est sensible, toujours justifiées par l'objet librement choisi, jamais considérées comme des techniques aveugles. L'adhésion à une équipe ne signifie pas la perte de la singularité de chacun, même si les membres s'organisent autour d'un projet commun.

Dans ce sens, si le corps pendant longtemps s'est trouvé hors de la question sociale, notamment dans l'éviction des relations entre le milieu naturel et les techniques corporelles, nos sociétés contemporaines pressées d'être et de paraître le marquent bien en le codant et le programmant, tout en provoquant des dynamiques réactionnelles qui tendent à montrer que les relations entre le corps et la culture ne se formulent pas uniquement dans une logique causaliste et déterministe. 
10 Tenter de comprendre ces questions posées au corps dans ses multiples dimensions, et non pas exclusivement sportives, c'est vouloir le lier aux transformations des contextes et aux variabilités de la culture, c'est peut-être vouloir le considérer aussi, comme "un analyseur transactionnel" (non pas au sens de Winnicot, c'est-à-dire comme objet permettant de supporter la séparation), produit autant par les cultures et les sociétés que participant à leur édification. C'est aussi ne pas se fermer à quelque perspective théorique dominante et unitaire - qui deviendrait vite terroriste de la pensée - ni à une science tellement "scientiste" qu'elle en oublierait de se centrer sur l'homme. C'est donc accepter le pluriel et ouvrir à toute approche permettant de mieux comprendre les rapports entre le corps et la culture appréciés dans ses réalités historiques ou contemporaines, éclairés par des disciplines comme l'anthropologie, l'ethnologie, la sociologie, l'histoire, la psychologie sociale, l'économie ou la philosophie et par des références au symbolique, à l'imaginaire et au pulsionnel, identifiés dans des objets comme le sport, la santé, le loisir, l'environnement, le milieu scolaire, les organisations sportives. Les objectifs viseront à capter les effets de la technoscience, de l'économique ou du politique, à repérer les mutations des valeurs sociales et les transformations des modes de vie des acteurs, à caractériser les adaptations des organisations générées par les renouvellements des goûts, à clarifier les enjeux politiques, économiques et symboliques des acteurs, à analyser la dialectique tradition-modernité liée aux permanences et aux mutations des pratiques corporelles, voire à établir des éléments de prévision rationnellement construits en matière de demandes sportives pour saisir leurs effets sur l'aménagement de l'environnement ou sur la qualité des services proposés. Dans un contexte général en constante turbulence, ces recherches seront également attentives à saisir les bouleversements épistémologiques dans les théories construisant la réalité sociale en montrant leurs conséquences concrètes sur la perception de la culture. Elles s'efforceront en outre de révéler les adaptations des acteurs et des institutions consécutives à l'émergence de nouveaux rapports au corps les marchés économiques concurrentiels s'appropriant le corps en déstructurant les anciens repères culturels - ou encore le rôle des politiques locales dans le développement sportif cherchant à satisfaire la multiplicité des goûts des usagers, ou enfin les innovations technologiques dérégulant la tradition et générant de nouvelles pratiques corporelles exploitées dans de nouveaux espaces remodelant de nouveaux "arts de faire", ou provoquant réactionnellement des réappropriations de la personne. Les articles présentés dans ce premier numéro pourraient paraître éclatés même s'ils sont réunis autour de thèmes qui les agrègent. Ils ne veulent signifier pour l'instant qu'une volonté de concrétisation d'axes de recherches qui se positionnent dans les directions définies par les membres du groupe organisés autour de ce projet commun dont les formes sont loin d'être définitives. Cette diversité consubstantielle au fondement de l'équipe qu'ils expriment doit donc s'entendre comme le résultat momentané de l'état d'esprit d'un groupe, moins soucieux de se positionner pour l'instant dans le complexe concurrentiel des S.T.A.P.S en se dotant immédiatement d'une image repérable, définitive et stable, que de signifier une existence et de poursuivre donc par cette production la logique d'institutionnalisation, en mettant en avant l'idée d'inachèvement et d'imprévu. Cette publication s'ordonne autour de quatre axes.

11 Le premier axe, "Thématiques", aborde trois questionnements justifiés par les problèmes relatifs aux transformations causées par le développement du sport ou par la place qu'il occupe dans diverses institutions : celui pour les organisations sportives 
de trouver des formes d'adaptation à la mouvance des goûts sportifs, celui pour les stages de réinsertion sociale d'apprécier la place du sport dans la redynamisation des sujets handicapés non préparés à le recevoir et enfin, celui de la recherche par les pratiquants d'une valeur comme le plaisir dont la variabilité des expressions doit être analysée. Concernant la première question, Jean-Pierre Chaze met en évidence les modifications qu'ont connues les organisations sportives municipales dans les dernières années pour en montrer leur complexification croissante nécessitée par la diversification de la demande, tandis que Charles Pigeassou s'attache à décrypter, au moyen d'une enquête par questionnaires, les représentations des dirigeants du mouvement sportif associatif pour en saisir les différences qui sont aussi autant d'enjeux pour faire face aux transformations qu'il repère dans le développement $d u$ sport contemporain. Tous deux posent le problème de ces transformations en terme de management. La deuxième question est abordée par Gilles Bui-Xuân, Anne Marcellini et Jacques Mikulovic qui renversent l'idée couramment admise selon laquelle la réinsertion professionnelle passe nécessairement par une formation ajoutée et qui montrent au contraire - relativement à une population de handicapés -, comment le sport, en introduisant un rapport à la corporéité peut, selon les sujets, contribuer à leur autonomisation dans la quête d'un emploi. La troisième question, celle du plaisir du pratiquant est soulevée par Guy Haye qui établit une revue de question sur un sujet paradoxalement délaissé par la recherche avant de proposer une analyse sur la capacité de la technique et de l'instrument à développer cette valeur dès lors qu'ils favorisent les capacités exploratoires du pratiquant ici repérées dans une activité de pleine nature au moyen d'une approche éthologique.

12 Le deuxième axe, "Études critiques", entend soulever des problèmes épistémologiques liés à la constitution de la connaissance sur le corps ou sur la culture dans le champ social et dans le champ scolaire. Cette épistémologie n'est jamais abstraite ni désincarnée mais contribue au contraire à montrer, de façon concrète, les conséquences des choix opérés dans ces champs et à proposer alors des alternatives. Dans ce sens, Jacques Gleyse prolonge sa réflexion sur l'axiologie sous jacente au développement des S.T.A.P.S et de l'E.P.S, en montrant comment ici la prise en compte $\mathrm{du}$ corps est minimisée au nom des normes scientifiques et universitaires. Ceci le conduit à rompre avec le sigle S.T.A.P.S dont il dissèque les composantes et à lui substituer le terme "anthropo-somatologie" centré sur une vision non scientiste de la corporéité. Cette conflictualité des savoirs scientifiques construisant les regards sur les pratiques corporelles est également abordée par Yves Le Pogam qui repère les antagonismes des théories sociologiques appréhendant le thème de la massification des pratiques sociales, dont les enjeux visent la reconnaissance ou non de ces pratiques comme pratiques "cultivées" ce qui n'est pas sans conséquences sur l'importance que l'école accordera à ces pratiques. Ici encore les problèmes nés des antagonismes théoriques dépassent largement les enjeux scientifiques "purs" qui sont aussi des positionnements sociaux dont on aurait tort de minimiser l'impact sur les changements qu'ils sollicitent dans la production ou dans la reproduction des pratiques sociales.

13 Le troisième axe est consacré à un auteur. Nous avons choisi pour ce numéro Pierre Sansot, Professeur d'Anthropologie à l'Université Paul Valéry de Montpellier, agrégé de philosophie. Ceux qui ont eu la chance de le rencontrer lors de colloques gardent de lui une image "physique", identifiée bien sûr par l'originalité d'une queue de cheval rompant avec le monde parfois austère de l'Université et des sciences, mais aussi l'image d'un universitaire aux capacités de communication exceptionnelles tenant à 
son humour, à la passion de ses propos et à ses dons peu communs d'observation des autres et sachant en outre faire resurgir l'essentiel de ce qui paraît insignifiant. Ce choix se justifie aussi par l'intérêt, bien plus, par la passion qu'un universitaire dont l'autorité est reconnue dans le complexe des sciences sociales en France, ose porter au sport. Pierre Sansot nous avait transmis quelques pages inédites dans lesquelles il dévoile, sous le mode du "je", cette alliance peu commune entre une sensibilité pour décrire "son" Languedoc et une écriture qui sait nous transmettre cette émotion en provoquant notre adhésion, nous bouleversant au sens où l'auteur nous fait découvrir par une approche audacieuse alliant l'imaginaire et le réel, une anthropologie dans laquelle la connaissance produite est le résultat heureux de cette combinatoire entre la subjectivité et une réalité irréductible alors à une "pure" objectivité. Yves Le Pogam tente ensuite de présenter quelques idées des approches si singulières de cet auteur qui se démarque des positionnements dominants dans les sciences sociales attentives aux faits de la vie quotidienne - dont les effets peuvent toucher aussi les théorisations du sport dans le champ des S.T.A.P.S. - sans avoir la prétention de restituer là toute la richesse des pages de l'auteur qui ne peut être appréhendée que par le contact avec son écriture originelle.

Enfin, un dernier axe "Notes de lectures", se propose classiquement de présenter quelques ouvrages, revues ou articles récents qui touchent au thème "Corps et culture" et qui s'intègrent de manière plus générale dans les directions de recherche de l'équipe.

\section{AUTEUR \\ YVES LE POGAM}

UFR STAPS Montpellier Equipe "Corps et Culture" 